\title{
DYNAMO ACTION, BETWEEN NUMERICAL EXPERIMENTS AND LIQUID SODIUM DEVICES
}

\section{JACQUES LÉORAT}

Observatoire de Paris-Meudon 92195 Meudon

P. LALLEMAND

ASCI, bat.506, Université Paris-Sud,91405 Orsay

J.L. GUERMOND

LIMSI, Université Paris-Sud,91405 Orsay

\author{
AND \\ F. PLUNIAN \\ LEGI, Institut de Mécanique, Université J. Fourier, Grenoble
}

\section{Introduction}

Conversion of kinetic energy into magnetic energy within a conducting fluid is but an example of various physical phenomena occuring in MHD turbulence at magnetic Reynolds numbers $R_{m}$ greater than a few tens. While the first numerical evidence of this phenomenon has been obtained since almost 45 years, it was only at the end of the year 1999 that it could be experimentally verified in liquid sodium flows. There is still a great gap between such experimental approaches and the understanding of natural dynamos, which are responsible for example of solar magnetic activity or of the geomagnetic field. To get closer to the real MHD problems, new designs of experimental devices and new numerical codes are needed in order to achieve greater $\mathrm{Rm}$, study the turbulent characteristics, the nonlinear saturation regime and the influence of large-scale flow configuration and boundaries.

Most numerical dynamos are based either on laminar or on low kinetic Reynolds number flows. Moreover, the magnetic Prandtl number, which is of the the order of $10^{-5}$ for liquid metals (Earth's case) is often chosen closer to unity in numerical simulations. Experimental fluid dynamos are needed because the spatial resolutions achieved in direct numerical simulations are unable to describe high kinetic Reynolds number flows which are the 
rule in astrophysical situations. While large eddy simulations or statistical models (such as EDQNM), leading to turbulent transport coefficients, could be usefull to describe fully developped turbulence, they must be validated using real MHD flow experiments. Finally, the exploration of the nonlinear saturation regime may be so expensive in computation time, as to prevent for instance the self consistent numerical study of an Earthlike chaotic dynamo or long magnetic cycles.

After presenting the energetic considerations of the experimental dynamo problem in the next Section, and comparing some driving configurations, we will examine the specific case of the precession driving using moderate Reynolds number simulations (Section 3). To prepare the design of a large scale precession experiment which will involve a fully turbulent flow, a water experiment has been designed and is planned to be in operation at the beginning of year 2001 (Section 4). The occurrence of the dynamo action and the non linear regime will be investigated with a numerical code convenient for cylindrical geometry (Section 5).

\section{Turbulence and energetics of a fluid dynamo}

There is no general sufficient condition for dynamo action which could be used as a guide for the selection of an efficient driving. Empirical computational experience shows that, in order to occur, dynamo action asks that the magnetic number of a given flow configuration be greater that a critical number $R_{m}^{*}$, which depends on the flow geometry. Positive numerical results are known either for "laminar dynamos", where the flow is at large scales and steady, or for "turbulent dynamos", where the velocity is fluctuating in space and time, with a zero mean. A real fluid dynamo cannot be based on a purely laminar (steady) flow nor on homogenous turbulence. However, to begin the feasibility study of an experimental fluid dynamo, it is usefull to ask first which dynamo model, laminar or turbulent, couldbe easier to realize, and which main parameters control the energy dissipation.

\subsection{TURBULENT DYNAMOS VERSUS LAMINAR ONES}

Different kinds of laminar dynamos are known in spheres, which is the simplest geometry for the numerical treatment of magnetic boundary conditions. In many cases, the corresponding $R_{m}^{\star}$ lies below 100 , which may be taken as a rough technical objective. Practical conditions for dynamo action in turbulent flows have not been investigated systematically, as they are generally obtained from non linear MHD computations which are rather demanding in numerical power. To the author's knowledge, they seem not to contradict the predictions of the EDQNM model for homogeneous MHD turbulence (vanishing mean velocity and helicity), where turbulent dynamo 
action occurs for turbulent magnetic Reynolds numbers $R_{m t}$ (based on $\mathrm{rms}$ speed and integral scale) greater than about 30 (Léorat et al 1981).

Injection of kinetic energy with zero mean is possible in principle using for example a sufficient number of turbines acting at a fraction of the container scale,. However, the maximal mean flow speed generated by a turbine will exceed the turbulent rms speed, so that one expects that, in practice, $R_{m t}<10 R_{m}$ (say), where the laminar magnetic Reynolds $R_{m}$ is based on the largest scale of the container and maximal speed of the mean flow: accordingly, when the turbulent dynamo could be excited,the laminar $R_{m}$ would be above 300 . This last number, whatever the crudeness of its estimation, is well above the critical threshhold $R_{m}^{\star}$ of many laminar dynamos.

Another look on this question may be obtained from turbulence phenomenology if non-local interactions in wavenumber space may be neglected (leading possibly to an inverse helicity cascade). In the Kolmogorov inertial range for the kinetic energy spectrum which extends for wavenumbers $k>k_{0}$, the local stretching rate $k u(k)$ scales as $k^{2 / 3}$, while the ohmic dissipation rate scales as $k^{2}$. The ratio between the two (which is a kind of local magnetic Reynolds number) scales as $k^{-4 / 3}$, and increases towards larger scales, starting from unity at the ohmic dissipation scale. For homogenous turbulence, it is maximal around the integral scale; while if a mean flow is present, it may continue to increase at larger scales. This argue again for the importance of the largest scales to achieve large magnetic Reynolds number flows.

Note also that in presence of a mean velocity flow, turbulence at smaller scales may favor dynamo action, as it happens that the ad-hoc introduction of small scales or a finite correlation time in laminar dynamos seems to reduce $R_{m}^{*}$ compared to the purely laminar case.

Turbulent dynamo action with a vanishing mean flow is certainly realized in some natural dynamos as the Sun, and is indeed also obtained in numerical experiments at large magnetic Prandtl numbers. It seems irrelevant for liquid sodium experiments (very small magnetic Prandtl number), where the main obstacle is the power constraint required to overcome the turbulent dissipation of kinetic energy.

\subsection{DRIVING POWER}

Liquid sodium, with its high electrical conductivity, seems the best fluid to be used in an experimental fluid dynamo. Many efficient numerical laminar dynamos are known to have a critical $R_{m}^{*}$ between 30 and 100 , and thus the kinetic Reynolds number of the flow will be around $10^{7}$. The flow which must be driven will be fully turbulent and a basic question of any exper- 
imental design involving such flows is to evaluate the amount of kinetic power needed to support the flow against viscous and ohmic losses. Assuming that the Kolmogorov cascade controls the non linear energy flux in the inertial range, and merging for simplicity turbulent and mean quantities (contrary to the preceeding sub- section), it is easy to obtain an expression for the power dissipated $P=k R_{m}^{3} / L$ where $\mathrm{k}$ is a coefficient characteristics of the geometry of the experiment (container, driving, etc...). The scaling of $\mathrm{P}$ with the Reyn olds number is experimentally verified, which supports the role of the Kolmogorov cascade in the flows driven by various means in a finite tank.

For a given available power, the scaling with $\mathrm{L}$ is in favor of large scale experiments, while the cubic scaling with $R_{m}$ would forbid to increase this number substantially. We want to stress here that the value of the power coefficient $\mathrm{k}$ should not be put aside in such a discussion. Its value is known (see these proceedings) in the following cases, say, $k_{R}$ (Riga), $k_{K}$ (Karlsruhe), $k_{C}$ (Cadarache), which may be compared with the case of pipe flow, $k_{P}$, where the turbulence level is minimal and which is not a candidate for dynamo action. For studies of some hydrodynamical features of the Cadarache experiment, see for example, Fauve et al 1993, Pinton and Labbé 1994 and Mordant et al 1997. From the published values of these experiments, one gets the following ordering, corresponding also to an ordering in turbulence levels:

$$
k_{R} / k_{P}=5, k_{K} / k_{P}=6.4, k_{C} / k_{P}=8.6
$$

This suggests that the path to larger magnetic Reynolds number flows has to be considered, using low $\mathrm{k}$ and large $\mathrm{L}$. While everybody knows how to increase $\mathrm{L}$ and $\mathrm{P}$, the search for lower $\mathrm{k}$ opens a new field : how store useful kinetic energy in a flow with the minimal turbulent dissipation ? "useful" is related to the occurence of dynamo action, which implies for example that any mean solid body rotation has to be substracted from the flow. As no sufficient condition for dynamo action is available, once a driving is selected on the basis of a low $\mathrm{k}$, the flow must be numerically tested with a MHD code. We will consider such a proposal below.

\section{Precession driving}

The interest towards flow generation at large scale without internal walls leads to consider driving the flow by the container wall itself, as occurs in the case of a precessing container. Upon the fast rotation of the container is superimposed a slow rotation (precession) at an angle with the rotation axis. For an arbitrary container shape, conservation of angular momentum of the fluid leads to a large scale circulation driven by pressure forces transmitted by the walls. In the case of the sphere, there is a peculiar inviscid 
solution due to Poincaré, with a transition to the wall through a viscous boundary layer. The case of the ellipsoidal container is interesting for the Earth dynamo: in this latter context, it has been first studied by Malkus (1968) and is still under active study (for France, cf studies at LGIT, Grenoble). An obvious technical advantage of precession driving for liquid sodium flows is the absence of seals on the container.

We propose to examine further the precession driving in the framework of experimental dynamos, using a cylindrical tank. This question has already been studied by Gans (1970), using a liquid sodium experimental device which gave torque measurements (and magnetic field), but without information on the actual flow configuration and was not developped further. To our knowledge, no related numerical simulations have been published on this topic.

The first issue which can be numerically tackled, at least for moderate Reynolds numbers flows, concerns the relative amount of toroidal and poloidal speeds which may be generated. Indeed, one of the few practical recipes derived from studies of linear numerical dynamos is that the ratio of these speeds should be close to unity to get optimized dynamos (it leads to efficient coupling between the magnetic field components). Simulations have been performed up to $R_{e}=5000$, using two different hydrodynamical codes, one based on lattice Boltzmann method (P. Lallemand, see d'Humières 1992) and the other on finite elements (Guermond and Quartapelle, 1997).

We consider a cylinder rotating around its symmetry axis and precessing around an axis orthogonal to the rotation one. The parameters of the problems are the aspect ratio (we choose heigth equal to diameter), the kinetic Reynolds number $R_{e}$ (defined as the inverse of the Taylor number ) and the precession rate, $p r$, i.e. the ratio of the precession to the rotation angular velocity. When $p r=0$, the steady flow is a solid body rotation and increasing $p r$ leads to an axial (poloidal) flow, accompanied by a decrease of the azimuthal velocity (see figure 1). At Reynolds numbers below about 2500 , the flow is steady, with central symmetry, while time fluctuations appear at larger Reynolds numbers, and the central symmetry is recovered only for the mean flow.

A first conclusion from this preliminary study is the generation of an axial speed close to $1 / 3$ of the container speed, and approximate equipartition of transverse and axial velocities which is obtained for $p r=0.1$, at least when the length of the container is equal to its diameter. The azimuthal energy decreases when the Reynolds number increases: this is a consequence of driving through viscous coupling with the cylindrical curved wall. If needed for dynamo action, more azimuthal energy could be generated inertially, using for example baffles fixed on both ends. 


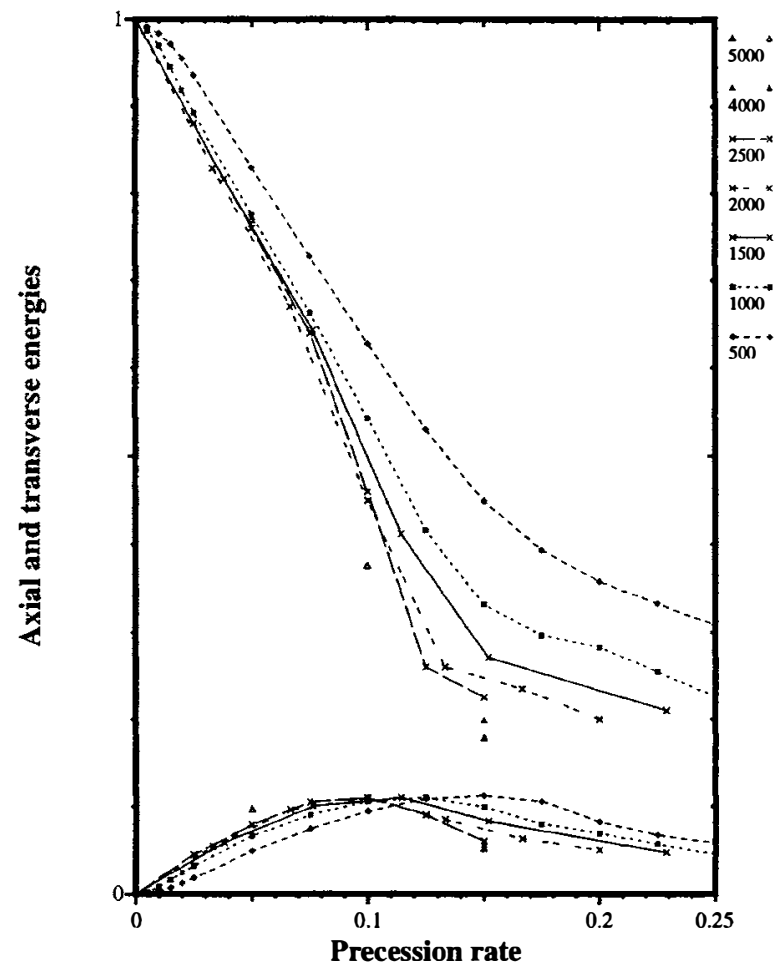

Figure 1. Variation with precession rate (pr between 0 and 0.25 ) of axial (lower curves) and transverse (upper curves) mean kinetic energies for different Reynolds numbers (between 500 and 5000). The energies are measured relatively to the solid body rotation energy

The numerical simulations cannot reach sufficiently large Reynolds numbers to produce a fully turbulent flow, from which the dissipated power and the power coefficient $k$ (see section 2.2 above) could be derived. These quantities may better be estimated using a water experiment which easily achieve Reynolds number above $10^{5}$, where asymptotic laws could be verified.

\section{Water experiment}

A water experiment has been designed and is currently under realization, with two main goals: 
(i) estimation of flow characteristics which could play a role in the design of a large scale experiment for the study of large $R_{m}$ flows using liquid sodium. This corresponds to practical questions such as the determination of the power coefficient $\mathrm{k}$ and the optimization of the precession rate and aspect ratio for dynamo action, with help of a numerical code (see below).

(ii) study of the influence of rotation on the larger scales of the turbulent flow. This more fundamental set of questions takes advantage of precession acting as a volume force on the flow, while the moving grids used generally in this context give rise to an absence of homogeneity of the turbulent features. This is why the device has been named ATER (agitateur pour la turbulence en rotation).

The tank is a fully transparent (PMMA) cylinder, with a diameter of 30 $\mathrm{cm}$ and a ratio of length/diameter which may be adjusted between $4 / 3$ and 1 . Is is rotating around its axis (horizontal) up to 10 turns/s, and precession about a vertical axis may be fixed up to $1 \mathrm{turn} / \mathrm{s}$. Velocities in the cylinder's sections will be obtained by particle image velocimetry (PIV), using a CCD camera corotating with the cylindrical container (optical axis coincide with cylinder axis) and a sheet of light orthogonal to the cylinder's axis and produced in a box fixed in the precessing frame.

\section{Numerical approaches}

Integration in time of the linear evolution equation of the magnetic field is used for the determination of the dynamo properties, as for example critical magnetic Reynolds numbers. Contrary to the eigenvalue method, direct simulations allows to study the consequences of time varying flows and time dependent external magnetic fields which may be used experimentally to estimate $R_{m}^{\star}$ from flow regime at lower $R_{m}$.

This approach has been used for example to optimize the Cadarache experiment (see Marié et al, these proceedings), with a code in cylindrical geometry, assuming axial periodicity of the flow and of the magnetic field. It has been verified that the conclusions were consistent with a more realistic, non periodic solution (F. Stefani, priv. communication), at least for the more important cases which could be compared.

The flow generated by precession is not compatible with axial periodicity: it leads to a strong poloidal circulation in a plane containing the rotation axis and orthogonal to the precession axis. Reproducing this flow by axial translation gives rise to a strong unrealistic shear on both ends of the container's ends. The use of a numerical code for MHD flows at large $R_{m}$ in a finite cylinder is thus required.

In order to be able to study the non linear MHD regime and to interpret experimental results, we have chosen to extend the existing hydrodynam- 
ical code based on a finite elements method, already used to study the precessing hydrodynamical flow. Another advantage of this code in relation with experimental devices is the easy implementation of spatial variations of conductivity of magnetic permeability of materials. The main question to consider first is the choice of the magnetic variables, electric and magnetic fields versus vector and scalar potentials, which are the most convenient to implement the boundary conditions relevant for an insolating external medium. To avoid the adjunction of a supplementary insolating envelope, the Laplace equation for the external scalar potential of the magnetic field may be solved with a surface element method (Guermond and Fontaine,1991). The relative merits of these approaches are currently examined using the Ponomarenko dynamo as a testing bench.

\section{Conclusion}

Dynamo studies have concentrated since a long time on the production of dynamos or antidynamo flows, leaving aside the complexities of the actual configurations of natural dynamos. The dynamo challenge has quite recently acquired new dimensions. After the first experimental evidences of dynamo action in laboratory, there is a need to design fluid dynamos at larger $R_{m}$ and without internal walls, and build MHD wind tunnels in order to study the nonlinear saturation regime under operating conditions closer to the ones of natural dynamos. The main obstacle to such experiments is a power constraint (kinetic energy injection and corresponding heat removal). MHD numerical codes adapted to the container geometry are also still needed to be able to predict the flow efficiency as a dynamo.

To make some progress in these directions, we propose to reexamine the case of precession forcing. With the $150 \mathrm{~kW}$ of electrical power which are needed to drive the Cadarache experiment $\left(R_{m}=70\right)$, if a driving design may be found such that its power coefficient is reduced by a factor 4 , say, using a container radius $\mathrm{L}=1 \mathrm{~m}$ (instead of $\mathrm{L}=0.2 \mathrm{~m}$ ) would lead to a flow at $R_{m}=190$. In the case of precession driving, assuming that the maximal speed of the flow is $1 / 3$ of the container rotational speed, this would correspond to a centrifugal pressure of about 33 atmospheres, which is quite technically feasible. The dynamo properties of this class of precessing flows remains an open question, to be tackled numerically.

\section{References}

1. d'Humières, D. (1992) Generalized lattice-Boltzmann equations in "Rarefied Gas Dynamics : Theory and Simulations", Progress in Astronautics and Aeronautics,159, B.D.Shizgal and D.P. Weaver edts, (AIAA, Washington, DC)

2. Fauve S., Laroche,C., and Castaing, B.,(1993) J.Phys.II,3,p 271 
3. Gans, R.F. (1970) On the hydromagnetic precession in a cylinder, J.Fluid. Mech.,45, pp 111-130

4. Guermond, J.L. and Quartapelle,L. (1997) Calculation of incompressible viscous flows by an unconditionally stable projection FEM, J. Comput. Phys. 132,pp 12-33

5. Guermond, J.L. and Fontaine,S. (1991) A discontinuous h-p Galerkin approximation of potential flows Rech. Aérosp. 4,pp 38-49

6. Léorat, J., Pouquet,A. and Frisch, U. (1981) Fully developped MHD turbulence near critical magnetic Reynolds number, J.Fluid. Mech.,104, pp 419-443

7. Malkus, W.V.R. (1968) Precession of the Earth as the cause of geomagnetism, Science, $\mathbf{1 6 0}$, pp 259-264

8. Mordant,N.,Pinton,J.F. and Chilla, (1997) J.Phys.II,7,p 1

9. Pinton,J.F., and Labbé, R.,(1994) J.Phys.II,4,pp 1461-1468 